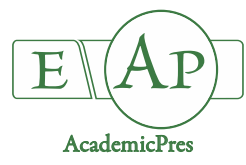

\title{
Interactive Effects of Plant Growth-Promoting Rhizobacteria and Phosphates Sources on Growth and Phosphorus Nutrition of Soybean under Moderate Drought
}

\author{
Vladimir ROTARU ${ }^{1 *}$, Luxita RISNOVEANU ${ }^{2}$ \\ ${ }^{1}$ Institute of Genetics, Physiology and Plant Protection, Mineral Nutrition and Water Regime of Plants Laboratory, 20 Pădurii Str., Chişinău, \\ MD-2002, Republic Moldova; rotaruvlad@yahoo.com (*corresponding author) \\ 2"Dunărea de Jos" University of Galati, Engineering and Agronomy Faculty of Brăila, Agronomy Center for Research and Consultancy and \\ Environment "Lunca", Brăila, Romania; luxita.risnoveanu@ugal.ro
}

\begin{abstract}
The growth of legume plants is usually improved by the rhizobacteria inoculation under low phosphorus (P) and alleviation of $\mathrm{P}$ nutrition plays important role in plant drought stress response. The aim of this study was to assess the comparative efficacy of two plant growth promoting rhizobacteria namely Burkholderia cepacia B36 and Enterobacter radicincitans $\mathrm{D} 5 / 23 \mathrm{~T}$ combined with two sources of phosphates in soybean (Glycine max L.) under low water supply. Plants were grown under $\mathrm{P}$ soluble versus insoluble $\mathrm{P}$ fertilization for comparing the effects of soybean inoculation on growth, uptake and use efficiency of phosphorus under moderate drought stress. At the beginning of flowering, half of plants was subjected to low water supply (35\% water holding capacity, WHC) for 12 days while control plants were well watered - 70\% WHC. The plants were harvested at the end of drought and physiological traits and $\mathrm{P}$ contents were analyzed. The inoculation treatments showed better plant growth and nutrient uptake when compared to uninoculated control. The application of the Burkholderia cepacia was more efficiently in terms plant growth than E. radicincitans especially under insoluble phosphates. Phosphorus concentrations of shoots and roots increased with both bacterial strains. The bacterial inoculation has much better stimulatory effect on nutrient uptake by soybean fertilized with insoluble phosphates. Study findings indicate that the combined application of PGPR (Burkholderia cepacia B36) and P amendments has the potential to improve P nutrition and growth of soybean cultivated on P-deficient soil under well-watered as well as moderate drought condition.
\end{abstract}

Keywords: drought; growth; phosphates; phosphorus nutrition; rhizobacteria; soybean

\section{Introduction}

Phosphate deficiency is a major constraint to plant productivity in many soils of the world, particularly in highly weathered soils of the humid tropics and acidic regions (Oberson et al., 2006). Large amounts of chemical fertilizers are necessary to provide normal growth and yield of crops. However, a large portion of soluble inorganic phosphate applied to soil as chemical fertilizer is immobilized rapidly after application due to phosphate fixation by aluminum, calcium, iron, magnesium and soil colloids (Rodriguez and Fraga, 1999) and becomes unavailable to plants (Merbach et al., 2010). Phosphorus chemical fertilizer is an expensive input and its use efficiency by crops may range from 10-25\% (Bahl and Singh, 1986). Natural rock phosphates and other $\mathrm{P}$ amendments have been recognized as valuable alternatives for $\mathrm{P}$ fertilizers, but nutrient efficiency of these sources is very poor. The assimilation of fixed soil $\mathrm{P}$ as well as hardly soluble phosphates using microorganisms is an option to augment the availability of $\mathrm{P}$ available form by the crops (Poonamgautam and Agnihotri, 2003). In agrosistems conditions, $\mathrm{P}$ deficiency often is accompanied by low soil moisture. The interactions between PGPR and phosphorus amendments could be a strategy in improving growth and nutrition of crops in different agroecosystems, particularly in P-deficient soils and water limited condition. Moreover, the use of beneficial soil microorganisms could reduce the 
amount of fertilizer input by increasing the efficiency of nutrient availability and other plant growth promoting activities (Adesemoye and Kloepper, 2009). Plant growth promoting rhizobacteria (PGPR) could effectively colonize plant roots and promote plant growth through various mechanisms such as increased mobilization of insoluble nutrients and thereby enhanced plant growth (Richardson et al., 2009; Esitken et al., 2010), increased nutrient availability in rhizosphere by producing siderophore (Kloepper et al., 1980), plant growth hormones (Aslantas and Çakmakçi, 2007; Khan et al., 2009; Egamberdieva et al., 2017a) and increased photosynthetic activity (Zhang et al., 1996). Several researchers have reported the ability of different bacterial species to solubilize insoluble inorganic phosphate compounds, such as tricalcium phosphate, hydroxyapatite and rock phosphate (Zaidi et al., 2003; Richardson and Richardson, 2011) and thereby increased the crop nutrition. Whitelaw (2000) demonstrated that the bacterial genera Pseudomonas, Bacillus, Rhizobium, along with Burkholderia (Fankem et al., 2006; Fernandez et al., 2007) are the most powerful P solubilizers. Likewise, $E$. radicincitans strain has ability to fix atmospheric $\mathrm{N}$ and produce phytohormones and other biological active substances (Ruppel et al., 2006).

Soybeans play an important role in sustainable agriculture because it is considered the major source of protein and vegetable oil as well as an essential amendment of soil fertility. However, this species is sensitive to P deficiency (Rotaru and Sinclair, 2009) as well as to drought (Jin et al., 2006). Therefore, is necessary to find out options to improve plant performance under such unfavorable environments. Krey et al. (2013) reported that the use of Pseudomonas and Enterobacter isolates increased P availability and corn growth under not limited water conditions. In addition, Garcia-Lopez et al. (2016) revealed that $\mathrm{P}$ availability in the medium is a crucial factor affecting benefits of the microorganisms on $\mathrm{P}$ uptake by plants. Unfortunately, there is scarce information regarding the comparison of the inoculation PGPR influences in combination with soluble or with hardly soluble phosphates application on crop performance under limited water supply. There are studies of growth promotion on crops plants inoculated with tested isolates in our experiments, but investigations performed under insufficiency water supply are missing for soybean plants. The relevance of such investigation is supported by the fact that both abiotic factors $\mathrm{P}$ deficiency and drought co-exist in field conditions. However, how $\mathrm{P}$ sources application together with the bacterial inoculation interacts to affect plant physiology and growth under limited soil moisture is poorly understood. The following hypothesis was tested: inoculations with PGPR bacteria and application of $\mathrm{P}$ amendments can increase plant growth and nutrient uptake under low water supply. Therefore, the present study was conducted to assess and compare the inoculation effect of two PGPR strains Burkholderia cepacia $\mathrm{B} 36$ and Enterobacter radicincitans $\mathrm{D} 5 / 23 \mathrm{~T}$ in the presence of soluble or insoluble phosphate source on growth and phosphorus uptake by soybean (Glycine max L.) grown in P-deficit soil and under moderate drought condition.

\section{Materials and Methods}

\section{Experimentaldesion}

Experiments were conducted in a greenhouse at Institute of Vegetable and Ornamental Crops (Grossbereen, Germany) under controlled conditions during 2013. The soil utilized in the experiment was acidic in reaction ( $\mathrm{pH} 5.5$ ) with initial Ct $1571 \mathrm{mg} / 100 \mathrm{~g}$ soil. Chemical properties of the soil were as follows: total N 173 $\mathrm{mg} / 100 \mathrm{mg}$, available phosphorus $2.5 \mathrm{mg} / 100 \mathrm{~g}$, potassium $10 \mathrm{mg} / 100 \mathrm{~g}, \mathrm{Mg} 3 \mathrm{mg} / 100 \mathrm{~g}$ soil, CN ratio 9:1. The not autoclaved soil was mixed with sand at proportion 1:1 (w:w). Soluble phosphate (monopotassium phosphate) and insoluble phosphate as calcium phosphate $\left(\mathrm{Ca}_{5} \mathrm{P}_{3} \mathrm{HO}_{13}\right)$ was uniformly mixed with the potted soil to supply $100 \mathrm{mg} P$ $\mathrm{kg}^{-1}$ soil. Calcium phosphate amendment $\left(\mathrm{Ca} \mathrm{P}_{3} \mathrm{HO}_{13}\right)$ has extremely poor solubility and it is denoted conventionally in the current paper as insoluble phosphate (IP). Basal macronutrients and micronutrients were applied in the soluble forms. The soil in all treatments was fertilized with $200 \mathrm{mg} \mathrm{K}\left(\mathrm{K}_{2} \mathrm{SO}_{4}\right), 100 \mathrm{mg} \mathrm{N}\left(\mathrm{NH}_{4} \mathrm{NO}_{3}\right), 20 \mathrm{mg} \mathrm{Mg}$ $\left(\mathrm{MgSO}_{4} \times 7 \mathrm{H}_{2} \mathrm{O}\right), 10 \mathrm{mg} \mathrm{Mn}\left(\mathrm{MnSO}_{4} \times 5 \mathrm{H}_{2} \mathrm{O}\right), 10 \mathrm{mg} \mathrm{Zn}$ $\left(\mathrm{ZnSO}_{4} \times 5 \mathrm{H}_{2} \mathrm{O}\right), 6 \mathrm{mg} \mathrm{Cu}\left(\mathrm{CuSO}_{4} \times 5 \mathrm{H}_{2} \mathrm{O}\right), 2 \mathrm{mg} \mathrm{B}$ $\left(\mathrm{H}_{3} \mathrm{BO}_{3}\right), 1 \mathrm{mg} \mathrm{Mo}\left(\mathrm{H}_{24} \mathrm{Mo}_{7} \mathrm{~N}_{6} \mathrm{O}_{24} \times 4 \mathrm{H}_{2} \mathrm{O}\right)$ and $10 \mathrm{mg} \mathrm{Fe}$ (Fe-EDTA) per $\mathrm{kg}$ soil to obtain a not nutrientinsufficiency substrate. All reagents used in the experiments and for plant and soil analyses were analytical grade (Sigma Aldrich).

\section{Plant growth}

Soybean (Glycine max L. cv. Zodiac) seeds were surface sterilized with $2.5 \%$ sodium hypochlorite, subsequently washed several times with sterile distilled water, and then germinated on the filter paper for 3 days at room temperature $\left(25-26{ }^{\circ} \mathrm{C}\right)$. Bacteria inoculation was performed by soaking the seedlings of soybean for $30 \mathrm{~min}$ within a freshly prepared suspension of Burkholderia cepacia B36 (denoted as B) and Enterobacter radicincitans D5/23T (denoted as E) with CFU $=10^{8} \mathrm{~mL}^{-1}$. Ruppel of the Department plant nutrition of Institute ornamental and vegetables plants (Grossbereen, Germany) offered these isolates. Six treatments were arranged including control soluble phosphate as $\mathrm{KH}_{2} \mathrm{PO}_{4}$ (denoted as $\mathrm{K}-\mathrm{P}$ ) without rhizobacteria inoculation; K-P inoculated with Burkholderia cepacia (denoted as K-P+B), K-P inoculated with Enterobacter radicincitans (denoted as $\mathrm{K}-\mathrm{P}+\mathrm{E}$ ), insoluble phosphate $\left(\mathrm{Ca}_{5} \mathrm{P}_{3} \mathrm{HO}_{13}\right)$ without inoculation (this insoluble $\mathrm{P}$ amendment was abbreviated as Ca-P), Ca-P inoculated with Burkholderia cepacia (denoted as $\mathrm{Ca}-\mathrm{P}+\mathrm{B}$ ) and $\mathrm{Ca}-\mathrm{P}$ inoculated with Enterobacter radicincitans (denoted as $\mathrm{Ca}-\mathrm{P}+\mathrm{E}$ ). One plant was grown in the middle of each pot. Because the Enterobacter has ability to fix $\mathrm{N}_{2}$ we did not treat the seeds with Bradyrhizobium japonicum to exclude its effect on nitrogen contents in plants. Thereafter the seedlings were grown in $3 \mathrm{~L}$ pots filled with sand-soil mixture. We use not autoclaved soil in order to perform study more adequate to field conditions. The soil used in experiments was disinfected by san's raises and in all treatments inclusive the control was used the same soil. The pots were distributed in a complete randomized block 
874

design with 5 replications. The pots were set up completely randomized in a greenhouse in Grossbereen, Germany (long. $13^{\circ} 19^{\prime} 60^{\prime \prime} \mathrm{E}$; lat. $51^{\circ} 22^{\prime} 0^{\prime \prime} \mathrm{N}$ ), and plants were grown for 6 weeks. Trials were performed in a temperaturecontrolled glasshouse with night/day temperatures of 20/26 ${ }^{\circ} \mathrm{C}$ and a $16 \mathrm{~h} \mathrm{~d}^{-1}$ photoperiod with complementary illumination. The relative air humidity averaged $65 \%$. During the first 30 days after sowing, water loss from the planting pots was estimated gravimetrically once per two days, and replaced with deionised water to maintain an average soil water content of approximately 70\% WHC. Thereafter, water supply was either maintained at this level (well-watered treatments, WW), or decreased to impose water stress (WS) at the 35\% WHC. At the beginning of the drought stress period plants were about to start flowering. For maintaining the low soil moisture level, the pots with plants were allowed to dry to the required level. After each day, the pots with the plants were weighed in the morning hours and the amount of water lost by evapotranspiration from the soil was replaced by watering the pots with the same volume of deionized water to bring the soil water status back to $70 \%$ and 35\% WHC.

Desired WHC of soil was maintained by irrigating after weighting the pots. Six control pots were kept without plants; three of them with 70\% WHC and three of them with dry $(35 \% \mathrm{WHC})$ soil to estimate evaporation from the surface of the soil. Low soil water regime was given for 12 days at flowering stage as sensitive stage to water deficit and after that, the plants were harvested. At harvest, shoots were cut and roots washed. They were oven-dried at $70{ }^{\circ} \mathrm{C}$ for 3 days, weighed and milled. After their DW was estimated, shoots and roots were analyzed for their P concentration separately.

\section{Plant analyses}

Phosphorus concentrations in the samples were analyzed calorimetrically with a spectrophotometer (EPOS 5060, Eppendorf, Hamburg, Germany) at $436 \mathrm{~nm}$ wavelength after staining with ammonium-molybdatevanadate solution (Gericke and Kurmies, 1952). The P uptake and $\mathrm{P}$ utilization efficiency was computed according to the methods reported (Kumar et al., 2014). Shoot biomass and root biomass $\mathrm{P}$ uptake per pot was calculated by multiplying biomass $(\mathrm{g})$ with $\mathrm{P}$ concentration $\left(\mathrm{mg} \mathrm{g}^{-1}\right)$. The $\mathrm{P}$ use efficiency (PUE) was calculated as the ratio of plant dry mass $(\mathrm{g})$ and plant $\mathrm{P}$ content $(\mathrm{mg})$. The stress tolerance index $(\mathrm{STI})$ was calculated as $S \mathrm{TI}=\mathrm{DW}_{\mathrm{w}} \times$ $\mathrm{DW}_{\mathrm{s}} / \mathrm{DW}^{2}{ }_{\mathrm{w}}$, where $\mathrm{DW}_{\mathrm{w}}$ and $\mathrm{DW}_{\mathrm{s}}$ were the dry weights under well-watered and drought conditions, respectively, and $\mathrm{DW}_{\mathrm{w}}$ was the mean dry weight under well-watered conditions.

Data on different parameters were statistically analyzed by using Statistic 7 program and mean differences among the treatments were compared by Least Significant Difference (LSD) test at $5 \%$ level of probability.

\section{Results}

Soybean growth was evaluated by measuring root dry weight, shoot dry weight, and total plant dry weight of inoculated and non-inoculated plants under two $P$ sources and two soil moisture levels at flowering (6 weeks after sowing) stage. The studied rhizobacteria stains (B. cepacia B36 and $E$. radicincitans $23 / 5 \mathrm{D})$ in conjunction with soluble or insoluble P fertilizations affected growth of soybean (Glycine max L.) under both normal soil moisture (control, $70 \%$ WHC) and moderate drought (35\% WHC) conditions (Table 1). The soybean seed inoculation with PGPR isolates increased dry weights of shoots of plants grown under low soil water regime, irrespective of $\mathrm{P}$ sources compared to uninoculated reference plants. Under nonlimited soil moisture, the highest dry weights of shoots (3.67 g plant ${ }^{-1}$ ) were recorded in soybean grown with soluble phosphorus in combination with B. cepacia inoculation. Examination of the shoot growth of plants subjected to low water supply (35\% WHC) also revealed a positive effect of this rhizobacteria species on dry matter production in presence of both P amendments. Drought stress had a pronounced negative effect in reducing shoot growth and less on root growth (Table 1).

The detrimental effect of drought was attenuate partially by PGPR inculcation compared with application of $\mathrm{P}$ amendments alone. The inoculation of soybean with $B$. cepacia strain in conjunction with soluble $\mathrm{P}$ application increased the shoot dry weight by $7.3 \%$ under normal water supply and by $19.6 \%(P<0.05)$ under moderate drought stress as compared to without inoculation treatment. Their influences were observed evidently on plants treated with insoluble phosphate. B. cepacia strain was most effective for growth stimulation under drought, where the dry matter of shoots and roots increased up to $55.4 \%$ and $26.7 \%$ respectively, compared to untreated plants. The application of $E$. radicincitans did not change significantly the dry matter production of shoots under well-watered conditions irrespective of P sources. However, this strain increased

Table 1. Effects of inoculation with B. cepacia and E. radicincitans on dry weights of shoots and roots of soybean plants fertilized with soluble or insoluble $\mathrm{P}$ sources in relation to water supply

\begin{tabular}{cccc}
\hline \multirow{2}{*}{ Treatments } & \multicolumn{2}{c}{ Shoot dry weight $\left(\mathrm{g} \mathrm{plant}^{-1}\right)$} & \multicolumn{2}{c}{ Root dry weight $\left(\mathrm{g}\right.$ plant $\left.^{-1}\right)$} \\
\cline { 2 - 4 } & WW-well-watered & WS- water stress & WW-well-watered \\
\hline K-P & $3.42 \pm 0.05$ & $2.04 \pm 0.012$ & $0.69 \pm 0.04$ \\
K-P+B & $3.67 \pm 0.12$ & $2.44 \pm 0.14$ & $0.72 \pm 0.10$ \\
K-P+E & $3.24 \pm 0.14$ & $2.43 \pm 0.05$ & $0.62 \pm 0.11$ \\
Ca-P & $0.90 \pm 0.07$ & $0.65 \pm 0.05$ & $0.41 \pm 0.03$ \\
Ca-P+B & $1.05 \pm 0.02$ & $1.01 \pm 0.03$ & $0.39 \pm 0.03$ \\
Ca-P+E & $0.96 \pm 0.09$ & $0.81 \pm 0.04$ & $0.38 \pm 0.03$ \\
\hline K-P: monopotassium phosphate uninoculated; + B: with application of Burkholderia cepacia B36; +E: with application of Enterobacter radicincitans D5/23T; Ca-P:
\end{tabular}


shoot growth by $19.1 \%$ in plants fertilized with soluble phosphate and by $24.6 \%$ with insoluble phosphate treatment under water stress conditions. In the current experiment, there was no significant difference between root dry weights of two isolates for soluble or insoluble $\mathrm{P}$ treatments under normal water soil regime. However, under water, limited conditions the application of rhizobacteria with insoluble phosphates increased root development and $B$. cepacia strain displayed better effect than $E$. radicincitans strain (Table 1). In comparison with IP applied alone, inoculation with $B$. cepacia in conjunction with this $\mathrm{P}$ source induced an increase in root growth of $27 \%$ and in treatment with Enterobacter radicincitans of $10 \%$ under moderate drought condition. Effects of rhizobacteria inoculation on total plant dry matter accumulation in relation to $\mathrm{P}$ sources and soil moisture levels are shown in Fig. 1A, B. The inoculation of soybean had different impact on dry matter production of plants cultivated with soluble phosphate as well as with insoluble phosphates. No significant differences on this parameter were observed between two tested bacteria strains in treatments with soluble phosphate. The effectiveness of PGPR in increasing plant growth was more relevant under insoluble phosphates and moderate drought conditions (Fig. 1B). The lowest plant production was registered in treatment with IP application without inoculation regardless soil moisture levels. However, inoculation with B. cepacia had more pronounced effect on plant growth than E. radicincitans strain.

Besides improvement of plant growth, these rhizobacteria species in combination with sources of phosphates exhibited influences on phosphorus contents in plant parts. The results on the phosphorus contents in shoots and roots affected by $\mathrm{P}$ amendments and bacterial inoculation are shown in Figs. 3 and 4. Contents of phosphorus in shoot and root tissues of soybean plants varied upon inoculation of rhizobacteria irrespective of soil moisture conditions. The beneficial effect of bacterial strains was observed in both well-watered and insufficiency water supply. In treatments with soluble P fertilization, the lowest nutrient concentration was obtained in uninoculated plants and subjected to water deficit with mean of 2.04 $\mathrm{mg} / \mathrm{g}$ dry weight. However, the inoculation with PGPR along with soluble $\mathrm{P}$ fertilization induced a significant increase by $27 \%$ and $32 \% \mathrm{P}$ concentrations in insufficient

A

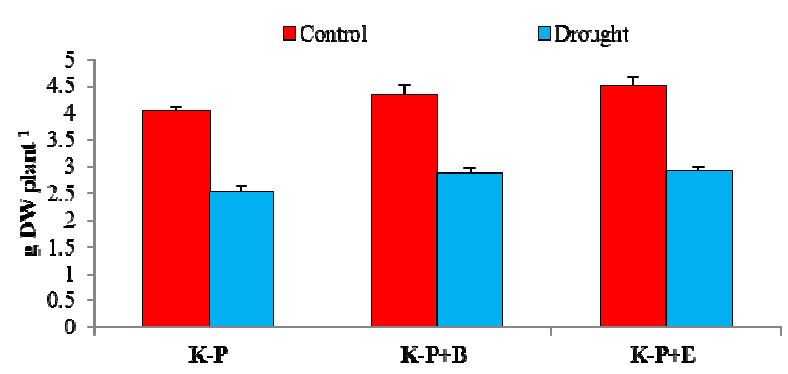

and sufficient water supply, respectively. Uninoculated plants exhibited very low shoot $\mathrm{P}$ concentration $(0.75-0.90$ $\mathrm{mg} / \mathrm{g}$ ) in treatment with insoluble phosphate application. Generally, P concentrations in shoots and roots increased significantly with soluble $\mathrm{P}$ fertilization in comparison to sparingly soluble $\mathrm{P}$ fertilization of plants regardless rhizobacteria species and soil moisture levels (Figs. 2 and 3). $\mathrm{P}$ contents in shoots of both rhizobacteria inoculations treatments were higher than in the uninoculated ones. Bacteria inoculation significantly increased $\mathrm{P}$ uptake while in contrast water stress significantly decreased it (Fig. 4A, B; $P<0.05)$. Likewise, it was observed that individual inoculation with $B$. cepacia or $E$. radicincitans produced significant increment in total $\mathrm{P}$ uptake of soybean plants compared to uninoculated control (Fig. 4). Much more pronounced effects were found out in case of plants receiving soluble $\mathrm{P}$ source and inoculated with $B$. cepacia. The beneficial influence of this strain on P uptake was also revealed in treatments with application of insoluble phosphate (Fig. 4B). Under unfavorable soil water condition, inoculation with B. cepacia in conjunction with soluble phosphates displayed better effect on $\mathrm{P}$ uptake by plants than bacteria $E$. radicincitans. In general, the least uptake of phosphorus was registered in uninoculated plants especially in treatment with application of insoluble form of phosphates alone. This trend was observed in both soil water regimes. Accordingly, the seed inoculation with Burkholderia sp. or Enterobacter sp. in combination with soluble source of $\mathrm{P}$ fertilization increased evidently nutrient uptake by plants. The magnitude of the increase in $P$ uptake due to inoculation with the B. cepacia ranged between $20 \%$ and $30 \%(P<0.05)$. In this trail, it was estimated the $P$ use efficiency by soybean in relation to rhizobacteria inoculation and forms of $\mathrm{P}$ amendments and experimental results are shown in Fig. 5. The P use efficiency (PUE) was affected by forms of $\mathrm{P}$ and less by rhizobacteria strains.

The experimental findings were shown that the combined use of strains with soluble phosphate had a weak trend to decrease this parameter irrespective of soil moisture level studied. Plants fertilized with IP and subjected to moderate drought registered an increasing of PUE in treatment with $E$. radicincitans in comparison to uninoculated plants fertilized with insoluble phosphate. The inoculation with that strain increased PUE of $11 \%$ and $13 \%$ under normal and drought conditions, respectively.

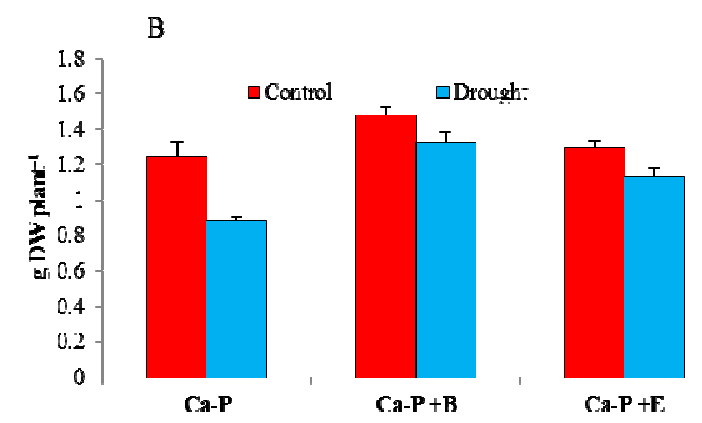

Fig. 1. Effect of soluble (A) or insoluble (B) P amendment applied alone or in combination with PGPR isolates on plant growth of soybean under well watered and drought conditions. Treatments: Ca-P, insoluble calcium phosphate; B: plants inoculated with Burkholderia cepacia; E: plants inoculated with Enterobacter radicincitans. Columns represent means for five plants $(n=5)$ with standard error bars 
876

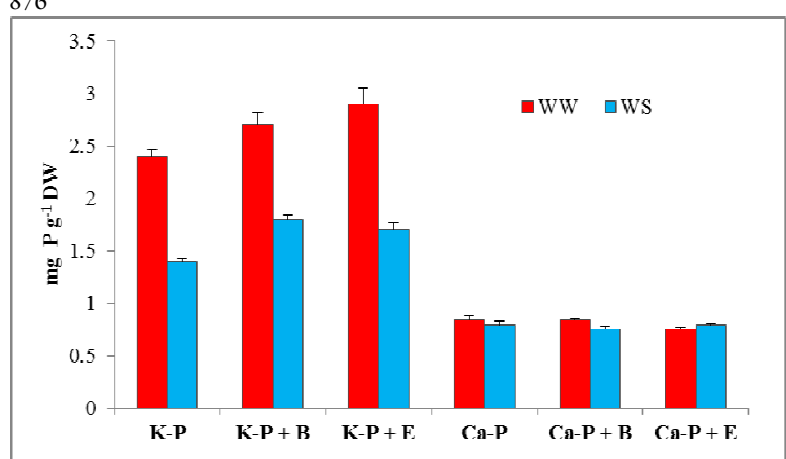

Fig. 2. Combined effect of plant growth-promoting rhizobacteria (PGPR) inoculation with soluble or insoluble phosphate on shoot $\mathrm{P}$ concentration of soybean under wellwatered (WW) and water stress (WS) conditions. Treatments: $\mathrm{K}-\mathrm{P}$, potassium phosphate; Ca-P, insoluble calcium phosphate; B: plants inoculated with Burkholderia cepaceia, E: plants inoculated with Enterobacter radicincitans. Vertical bars indicate the mean $(n=5) \pm S . E$

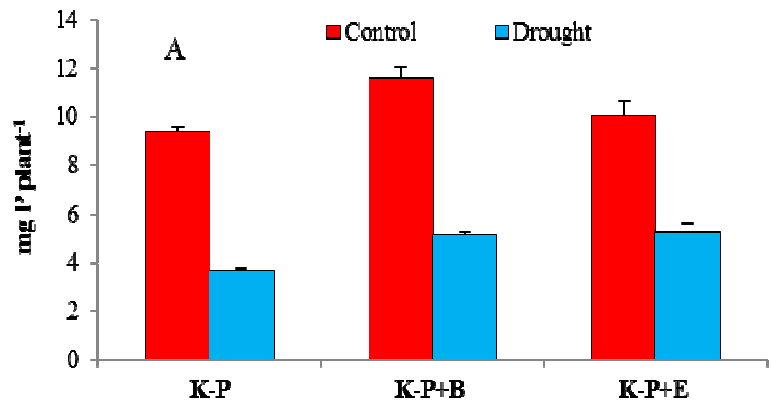

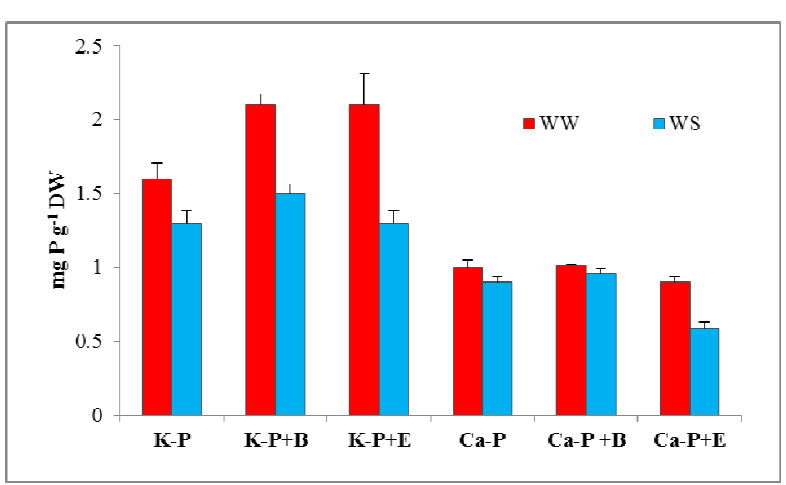

Fig. 3. Combined effect of plant growth-promoting rhizobacteria (PGPR) inoculation with soluble (K-P) or insoluble (Ca-P) phosphate on root $\mathrm{P}$ concentration of soybean under well-watered (WW) and water stress (WS) conditions. Treatments: K-P, potassium phosphate; Ca-P: insoluble calcium phosphate; B: plants inoculated with Burkholderia cepaceia, E: plants inoculated with Enterobacter radicincitans. Vertical bars indicate the mean $(n=5) \pm$ S.E

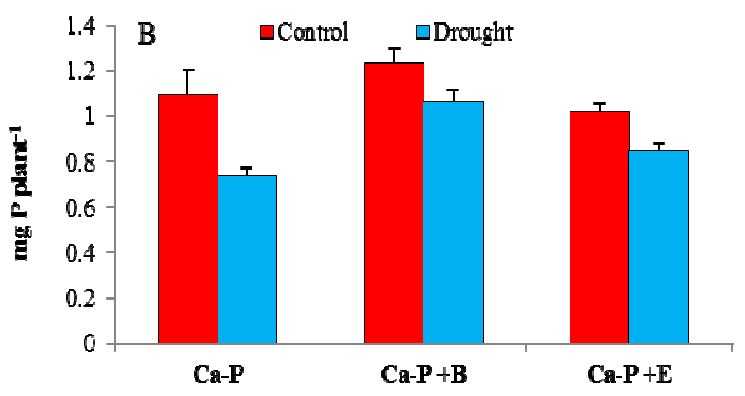

Fig. 4. Interactive effect of PGPR inoculation with soluble (A) or with insoluble P source on phosphorus uptake of soybean under well-watered and drought conditions. Treatments: K-P, potassium phosphate; Ca-P, insoluble calcium phosphate; B - plants inoculated with Burkholderia cepaceia, E - plants inoculated with Enterobacter radicincitans. Vertical bars indicate the mean $(\mathrm{n}=$ 5) \pm S.E

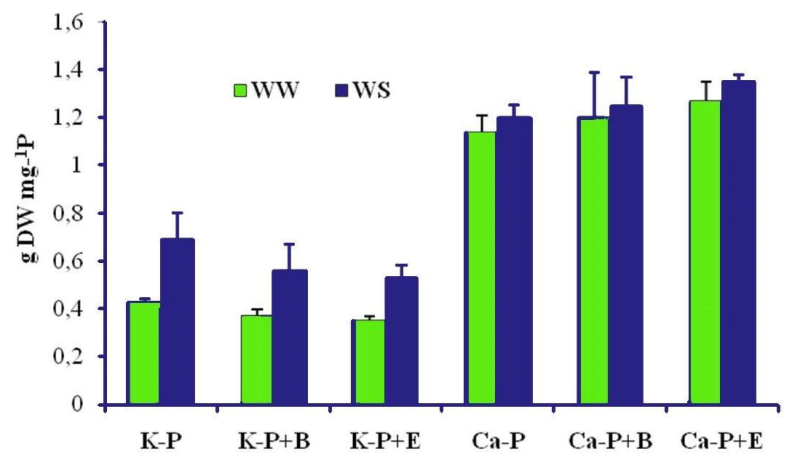

Fig. 5. Phosphorus utilization efficiency (PUE) of soybean following the application of different $\mathrm{P}$ sources applied alone or in combination with PGPR isolates under well-watered (WW) and water stress (WS) conditions. Treatments: K-P, potassium phosphate; Ca-P, insoluble calcium phosphate; $\mathrm{B}$ : plants inoculated with Burkholderia cepaceia, E: plants inoculated with Enterobacter radicincitans. Vertical bars indicate the mean $(n=5) \pm$ S.E.
The response of PUE to rhizobacteria was more pronounced when inoculants were used with insoluble $\mathrm{P}$ amendment. The current study demonstrated that inoculation with PGPR isolates and P sources interactively improved drought tolerance of soybean (data not shown). The increase of stress tolerance index (STI) was greater in plants grown with combined application of B. cepacia and insoluble $\mathrm{P}$ fertilizer compared to treatment without bacteria inoculation. The use $E$. radicincitans also improves the STI but at less extent compared to treatment of $B$. cepacia inoculation.

\section{Discussion}

The bacterial species have been investigated for their plant growth abilities of crops fertilized mostly with rock phosphates under normal water soil conditions (Kloepper et al., 1980; Gull et al., 2004; Maghraoui et al., 2016). The responses of crop growth and mineral nutrition to PGPR inoculation could be affected by adverse environmental conditions, especially by low soil water regime and P supply. 
In the current work, it was investigated whether two strains $B$. cepacia $36 \mathrm{~B}$ and $E$. radicincitans $23 \mathrm{D} / 5$ have ability to promote growth and improve $\mathrm{P}$ nutrition of soybean plants in presence of soluble or insoluble phosphates in relation to soil moisture level. Experimental results revealed that the growth and $\mathrm{P}$ contents in shoots and roots of soybean cultivated in P-deficit soil were affected by rhizobacteria strains and $\mathrm{P}$ amendments. However, the responses of soybean growth to inoculation with $B$. cepacia or $E$. radicincitans in relation to $P$ sources and soil moisture levels were different. It was observed that the vegetative growth (shoot and root dry weights, total plant weight) of plants under non-limited moisture was increased significantly after inoculation of seeds only with Burkholderia cepacia isolate. Its beneficial impact was related to $P$ solubilization capacity and production of phytohormones as reported in the literature. In general, the application of hardly soluble phosphates decreased significantly dry weights of shoots compared to plants fertilized with soluble $\mathrm{P}$ amendment. Similarly, low water supply diminished shoots and roots growth irrespective of P sources compared to well-watered ones. In addition, the shoot growth was decreased essentially in plants subjected to drought in treatments with insoluble phosphate application compared to those with soluble phosphate. Similar results were found out in the study of Zaidi et al. (2003) on chickpea but under normal soil moisture conditions. In the current study, inoculation of soybean with the PGPR isolates partially reduced negative effect of drought on plants. The application of Enterobacter sp. strain promoted growth of plants under IP and subjected to drought condition in comparison to IP alone. However, this strain did not affect significantly plant growth under normal moisture conditions. The higher reductions of shoots and roots growth were caused by combined effects of scarce conditions of $\mathrm{P}$ nutrition (treatments with IP) and low soil moisture. The ameliorative effects of different PGPR species on plant growth under drought conditions have been shown on various crops such as lupine (Egamberdieva et al., 2017b), common bean (Elkoca et al., 2010), chickpea (Gull et al., 2004) and strawberry (Esitken et al., 2010). Results of current trail are consistent with the findings of Egamberdieva et al. (2013) for which inoculation of Galega officinalis with Pseudomonas sp. improved growth under stress saline condition. Likewise, it was reported that combined application of PGPR (Bacillus subtilis QST713) with RP increased P uptake and growth of cucumber plants cultivated on P-deficit soil (Garcia-Lopez et al., 2016).

Data related to root growth of soybean in relation to PGPR inoculation and P forms under low water supply is limited (Abbasi et al., 2011). The changes of soybean root morphology and architecture under inoculation of beneficial microbes are essential in ability of nutrient acquisition by plants under unfavorable environments as was stated by Egamberdieva et al. (2017a). Root dry biomass production of soybean was determined to test its responses to PGPR application in relation to sources of phosphorus under normal and low water regimes of soil. Altogether, the increases of root dry weights by inoculation of soybean with added insoluble $\mathrm{P}$ source were greater than those in treatments with their inoculation in conjunction with soluble phosphate. Abiotic stress inhibits root growth and development, therefore decreasing plant ability to acquire nutrients and water from soil. Perhaps, the higher level of available $\mathrm{P}$ in soil reduced root colonization by PGPR and therefore their beneficial impact on plants was weak. Such kinds of plant growth responses to rhizobacteria inoculation under soluble P supply were denoted for other crop. The comparative efficiency of rhizobacteria strains demonstrated that Burkholderia sp. was most effective for plant growth promotion with sparingly soluble phosphate, where the total plant dry matter of soybean increased up to $54.4 \%$ under insufficiency water supply (35\% WHC) and of $26.7 \%$ in well-watered plants (70\% WHC). Enhanced growth was determined not only by amelioration of plant nutrition due to rhizobacteria application, we must take in consideration the possibility of the involvement of nitrogen assimilation and secretion of phytohormones, phosphatases, organic acids and other microbial activities by inoculants for promotion of growth and uptake of macronutrients and micronutrients (Islam and Hossain, 2012). These findings have also been reported by several researchers while investigating the inoculation effect of others species of phosphate solubilizing bacteria on a variety of crops (Zaidi and Khan, 2005). Likewise, Egamberdieva et al. (2017a) demonstrated that combined use of Bradyrhizobium japonicum and the entophyte Stenotrophomonas rhizophila increased growth and nutrients uptake of soybean under salt stress due to improvement of root architecture.

The association between PGPR and plant roots plays an important role in $\mathrm{P}$ nutrition in many agroecosystems, particularly in P-deficient soils. The mechanisms underlying the improved nutrient uptake, especially those nutrients of low mobility, e.g. P for PGPR inoculated plants under normal soil moisture have been documented (Dimkpa et al., 2009). In the present experiment, the interaction of bacterial inoculation along with soluble or insoluble $\mathrm{P}$ source affected nutrient contents in soybean tissues as compared to without inoculation treatments. The analyses of experimental results allowed suggesting that the beneficial effect of rhizobacteria on plant growth was attributed to efficiency of $\mathrm{P}$ uptake from soil and fertilizers. It is necessary to be mentioned that under low water supply, promotion of nutrient uptake is of greater importance because mobile nutrients become unavailable to plants due to the lack of solvent (Cui and Nobel, 1992). Similar to the present study' results, the increased nutrients uptake of both sparingly soluble and well soluble sources of $\mathrm{P}$ by microorganisms under adequate water regime have been documented for Vicia faba (Maghraoui et al., 2016). The nutrient uptake by soybean was reduced by small roots as was shown in current study, especially under insoluble $\mathrm{P}$ source, probably, associated with low physiological activity of plants subjected to insufficient water supply. According to current experimental data, higher $\mathrm{P}$ accumulation in shoots and roots of inoculated plants as compared to uninoculated ones were due to better roots development. The obtained results indicated that PGPR inoculation of soybean plants significantly increased $\mathrm{P}$ accumulation in shoots and roots compared to their reference treatments without inoculation. These findings are in agreement with data obtained by Xiurong et al. (2011). Experimental results revealed that much more pronounced effects on nutrient acquisition were found out in case of plants inoculated with 
878

B. cepacia. It was suggested that rhizobacteria application could increase root surface as well as roots hairs that contributed to better P acquisition efficiency by plants. In addition, some rhizobacteria species have ability to produce siderophore as well as axuins that contribute to increase acquisition capacity of plants for other nutrients from growth media. In the current study, the inoculation with PGPR along with soluble $\mathrm{P}$ fertilization induced a significant increase by $27 \%$ and $32 \% \mathrm{P}$ concentrations in limited and sufficient water supply, respectively. Probably, Fernandez et al. (2007) have demonstrated these bacteria strains enhanced plant nutrition due to their abilities to improve interactions between roots and microorganisms as. Meanwhile, these rhizobacteria enhanced total plant matter production which had a higher demand for nutrients including P. Likewise, increased P uptake was also found of both rice and wheat when PSB were applied with rock phosphates (Sharma et al., 2013) as well as within soybean by combined application of Bacillus sp. and RP under nolimited water supply (Fankem et al., 2014). Therefore, we agree with the proposition that rhizobacteria contribute to increase the roots surface area and the number of finite roots which had beneficial impact on plant capacity to P uptake. As we expected, plants grown under insoluble phosphate displayed a small concentration of the nutrient in vegetative tissues irrespective of inoculation treatments. Concerning the influence of $E$. radicincitans on soybean, analyses of experimental results allowed to make a supposition that the application of this strain had no significant effect on the plant growth, but exhibited a positive impact on $\mathrm{P}$ concentration in plants tissues. Zaidi and Khan (2005) also reported that colonization of plant roots by PGPR greatly increased the wheat capacity to nutrient uptake. Therefore, the results showed that there was a positive interaction of bacteria inoculation with $\mathrm{P}$ fertilization in promoting soybean growth and $\mathrm{P}$ uptake. The higher $\mathrm{P}$ acquisition rate was observed after application of the B. cepacia strain compared to the $E$. radicincitans strain and the results may indicate that $B$. cepacia is more effective in induction of plant ability for P uptake as well as other promoting biological activities. Similarly, inoculation with $P$ solubilizing $B$. megaterium, $\mathrm{N}_{2}$-fixing $B$. subtilis and Rhizobium leguminosarum bv. phaseoli caused a significant improvement of seed yields and a concomitant increase uptake of macronutrients and micronutrients by common bean (Elkoca et al., 2010). From a practical consideration, the increase of fertilizer effectiveness with the inoculation of beneficial microorganisms may help to reduce the doses of $\mathrm{P}$ fertilizers and to increase fertilizer efficiency (Pradhan et al., 2017). In particular, P solubilizing bacteria are reported to play a significant role in increasing the P efficiency of both native and applied $\mathrm{P}$ and improving the growth and yield of various crops (Khan et al., 2009). From this reason we hypothesized that integrated use of phosphate amendments with seed PGPR inoculation could be a promising option to increase the efficiency of phosphorus by soybean. In the current investigation, the lowest PUE was obtained in plants fertilized with soluble P fertilizer alone or in combination with rhizobacteria isolates. The inoculation with both rhizobacteria strains in presence of soluble phosphate had no effect on PUE in soybean regardless of soil moisture level. However, this parameter was increased in treatments with PGPR in conjunction with insoluble phosphates. Low plant matter production in response to IP source was, probably, one of the reasons for such effect. The combined application of $E$. radicincitans with IP increased this parameter compared to treatment without inoculation under moderate drought condition. A strong correlation between crop yield and mineral concentrations are widely known effect caused by insufficiency nutrient and water supply (Jarrell and Beverly, 1981). The role of PGPR in improving P utilization from applied P fertilizers has been reported by others researchers (Abbasi et al., 2011).

The rhizobacteria and $\mathrm{P}$ sources interaction is believed to be involved not only in the amelioration of plant mineral nutrition, but also in withstanding stress tolerances, such as drought. There is scarce information and conflicting reports exist in the literature regarding the influence of rhizobacteria in conjunction with soluble or insoluble phosphates on tolerance of soybean to drought. To our knowledge, the current study demonstrated for the first time that inoculation with tested PGPR isolates and P sources interactively affected this parameter under moderate drought. The use $E$. radicincitans enhanced stress tolerance index (STI). However, in this aspect B. cepacia displayed better results. The study's results corroborated findings of Gusain et al. (2015) with Pseudomonas strain inoculation of rice. The improvement growth and tolerance of plants to unfavorable conditions is mediated by many mechanisms induced by PGPR. One of these mechanisms is increasing nutrients uptake by plants, particularly in P-deficits soils as was found in the present investigation. Shen et al. (2011) have stated that appropriately manipulating P supply can enhance plant growth, nutrient uptake and the ability to resist various stresses, including water deficit. Some researches (Jin et al., 2006) and in our previous study (Rotaru and Sinclair, 2009) also demonstrated that the improvement of $\mathrm{P}$ nutrition due to phosphorus supplementation, increased soybean performance under drought. We proposed that enhanced soybean tolerance to both abiotic factors low $\mathrm{P}$ and moderate drought was caused partially by improving nutrition and growth as result of the use PGPR strains. Of course, there were other mechanisms induced by rhizobacteria contributing to promote a better capacity of soybean growth and development under unfavorable conditions. Thus, the interactions between PGPR and phosphorus amendments could be a strategy in improving crop productivity and tolerance to unfavorable environmental conditions, particularly in P-deficient soils and moderate drought conditions.

\section{Conclusions}

The results obtained from this comparative study suggest that plant growth stimulating efficiency of bacterial inoculants was affected by P sources and water soil regime. Integrated application of $\mathrm{P}$ sources and inoculation with PGPR (Burkholderia cepacia and Enterobacter radicincitans) improved P nutrition and growth of soybean plants under normal as well as moderate drought conditions. The 
bacterial inoculation showed better stimulating effect on the growth and nutrient uptake by soybean under insoluble $\mathrm{P}$ amendment application. The application of the B. cepacia was more efficiently in terms of plant growth and drought tolerance than $E$. radicincitans.

\section{Acknowledgements}

The research was supported by the DAAD (Germany) (nr. A|13|03092).

\section{References}

Abbasi MK, Mansha S, Rahim N, Ali A (2011). Agronomic effectiveness and phosphorus utilization efficiency of rock phosphate applied to winter wheat. Agronomy Journal 105(6):1606-1612.

Adesemoye AO, Kloepper JW (2009). Plant-microbes interactions in enhanced fertilizer use efficiency. Applied Microbiology and Biotechnology 85(1):1-12.

Aslantas RR, Çakmakçi FS (2007). Effect of plant growth promoting rhizobacteria on young apple tree growth and fruit yield under orchard conditions. Scientia Horticulturae 111(4):371-377.

Bahl GS, Singh NT (1986). Phosphorus diffusion in soils in relation to some edaphic factors and its influence on P uptake by maize and wheat. Journal of Agricultural Sciences 107(2):335-341.

Cui M, Nobel PS (1992). Nutrient status, water uptake and gas exchange for three desert succulents infected with mycorrhizal fungi. New Phytologist 122(4):643-649.

Dimkpa C, Weinand T, Asch F (2009). Plant-rhizobacteria interactions alleviate abiotic stress conditions. Plant Cell and Environment 32(12):1682-1694.

Egamberdieva D, Wirth S, Jabborova D, Räsänen LA, Liao H (2017a). Coordination between Bradyrhizobium and Pseudomonas alleviates salt stress in soybean through altering root system architecture. Journal of Plant Interactions 12(1):100-107.

Egamberdieva D, Reckling M, Wirth S (2017b). Biochar-based Bradyrbizobium inoculum improves growth of lupin (Lupinus angustifolius L.) under drought stress. European Journal of Soil Biology 78:38-42.

Egamberdieva D, Berg G, Lindström K, Räsänen LA (2013). Alleviation of salt stress of symbiotic Galega officinalis L. (goat's rue) by co-inoculation of rhizobium with root colonizing Pseudomonas. Plant and Soil 369(12):453-465.

Elkoca E, Turan M, Dönmez M (2010). Effects of single, dual and triple inoculations with Bacillus subtilus, Bacillus megaterium and Rhizobium leguminosarum bv. phaseoli on nodulation, nutrient uptake, yield and yield parameters of common bean (Phaseolus vulgaris L. cv. 'Elkoca-05'). Journal of Plant Nutrition 33(14):21042119.

Esitken A, Yildiz HE, Ercisli S, Donmez M, Turan M, Gunes A (2010). Effects of plant growth promoting bacteria (PGPB) on yield, growth and nutrient contents of organically grown strawberry. Scientia Horticulturae 124(1):62-66.

Fankem H, Nwaga D, Deubel A, Dieng L, Merbach W, Etoa FX (2006). Occurrence and functioning of phosphate solubilizing microorganisms from oil palm tree (Elaeis guineensis) rhizosphere in Cameroon. African Journal of Biotechnology 5(24):2450-2460.

Fankem H, Ghislain N, Laurette N, Tchuisseu T, Nwaga D, Etoa FX (2014). Rock-phosphate solubilising bacteria and their effect on soybean (Glycine max) growth under pot grown conditions. American Journal of Agriculture and Forestry 2(4):100-109.

Fernandez L, Zalba P, Gomez MA, Sagordoy MA (2007). Phosphatesolubilization activity of bacterial strains in soil and their effect on soybean growth under greenhouse conditions. Biology and Fertility of Soils43(6):805-810.

Garcia-Lopez AM, Avile M,Del A (2016).Effect of various microorganisms on phosphorus uptake from insoluble Ca-phosphates by cucumber plants. Journal of Plant Nutrition and Soil Sciences 179(4):454-465.

Gericke S, Kurmies B (1952). Die colorimetrische Phosphorsäurebestimmung mit Ammonium-Vanadat-Molybdat und ihre Anwendung in der Pflanzenanalyse. Journal of Plant Nutrition and Soil Sciences 159:11-21.

Gull M, Hafeez FY, Saleem M, Malik A (2004). Phosphorus uptake and growth promotion of chickpea by co-inoculation of mineral phosphate solubilizing bacteria and a mixed rhizobial culture. Australian Journal of Experimental Agriculture 44(6):623-628.

Gusain YS, Kamal R, Mehta CM, Singh US, Sharma AK (2015). Phosphates solubilizing and indole-3-acetic producing bacteria from the soil of Garhwal Himalya aimed to improve the growth of rice. Journal of Environmental Biology 36(1):301-307.

Islam MT, Hossain M (2012). Plant probiotics in phosphorus nutrition in crops, with special reference to rice. In: Maheshwari DK(Ed). Bacteria in Agrobiology: Plant Probiotics. Berlin, Springer,pp 325-363.

Jarrell WM, Beverly R (1981). The dilution effect in plant nutrition studies. Advances in Agronomy 34(1):197-224.

Jin J, Wang GH, Liu XB, Pan XW, Herbert SJ, TangC (2006). Interaction between phosphorus nutrition and drought on grain yield, and assimilation of phosphorus and nitrogen in two soybean cultivars differing in protein concentration in grains. Journal of Plant Nutrition 29(8):1433-1449.

Khan AA, Jilani G, Akhter MS, Naqvi SMS, Rasheed M (2009). Phosphorous solubilizing bacteria; occurrence, mechanisms and their role in crop production. Journal of Agriculture and Biological Sciences 2(1):48-58.

Kloepper JW, Leong J, Teintze M, Schroth MN (1980). Enhanced plant growth by siderophores produced by plant growth-promoting rhizobacteria. Nature 286(5776):885-886.

Krey T, Vassilev N, Baum C, Eichler-Löbermann B (2013). Effects of longterm phosphorus application and plant-growth promoting rhizobacteria on maize phosphorus nutrition under field conditions. European Journal of Soil Biology 55:124130.

Kumar A, Choudhary CS, Paswa D, Kumar B, Arun A (2014). Sustainable way for enhancing phosphorus efficiency in agricultural soils through phosphate solubilizing microbes. Asian Journal of Soil Science and Plant Nutrition 9(2):300-310.

Maghraoui T, Bechtaoui N, Galiana A, Wahbi S, Duponnois R, ... Oufdou $\mathrm{K}$ (2016). Effect of inoculation by Moroccan rock phosphatesolubilizing rhizobia, versus phosphorus fertilization, on the growth and 
880

the phosphorus uptake by Vicia faba. Pakistan Journal of Agricultural Sciences 53(4):817-826.

Merbach W, Deubel A, Gransee A, Ruppel S, Klamroth A-K (2010). Phosphorus solubilization in the rhizosphere and its possible importance to determine phosphate plant availability in soil. A review with main emphasis on German results. Archives of Agronomy and Soil Science 56(2):119-138.

Naiman AD, Latrónico A, Salamone de IEG (2009). Inoculation of wheat with Azospirillum brasilense and Pseudomonas fluorescents: impact on the production and cultural rhizosphere microflora. European Journal of Soil Biology 45(1):4451.

Oberson A, Bünemann EK, Friesen DK, Rao IM,Smithson PC, ... Frossard E (2006). Improving phosphorus fertility in tropical soils through biological interventions. In: Uphoff $\mathrm{N}$ et al. (Eds). Biological approaches to sustainable soil systems. CRC Press, Boca Raton FL, pp 531-546.

Poonamgautam AK, Agnihotri LM (2003). Effect of phosphorus rate and Pseudomonas species in combination with Bradyrbizobium japonicum and farmyard manure on seed yield and yield attributes of soybean (Glycinemax). Indian Journal of Agricultural Sciences 73(8):426-438.

Pradhan M, Kumar SR, Pradhan C, Tuteja N, Mohanty S (2017). Contribution of native phosphorous-solubilizing bacteria of acid soils on phosphorous acquisition in peanut (Arachis hypogaea L). Protoplasma 254(6):2225-2236.

Richardson AE, Richardson JS (2011). Soil microorganisms mediating phosphorus availability. Update on microbial phosphorus. Plant Physiology 156(3):389-396.

Richardson AE, Barea JM, McNeill AM, Prigent-Combaret C (2009). Acquisition of phosphorus and nitrogen in the rhizosphere and plant growth promotion by microorganisms. Plant and Soil 321(1-2):305339.
Rodriguez H, Fraga R (1999). Phosphate solubilizing bacteria and their role in plant growth promotion. Biotechnology Advances 17(45):319-339.

Rotaru V, Sinclair T (2009). Influence of plant phosphorus and iron concentrations on growth of soybean. Journal of Plant Nutrition 32(9):1513-1526.

Ruppel S, Rühlmann J, Merbach W (2006). Quantification and localization of bacteria in plant tissues using quantitative real-time PCR and online emission fingerprinting Plant and Soil 286(1-2):21-35.

Sharma SB, Sayyed RZ, Trivedi MH, Gobi TA (2013). Phosphate solubilizing microbes: sustainable approach for managing phosphorus deficiency in agricultural soils Springerplus 2(1):587-611.

Shen J, Yuan L, Zhang J, Li H, Bai Z, ... Zhang F (2011). Phosphorus dynamics: from soil to plant. Plant Physiology 156:997-1005.

Whitelaw MA (2000). Growth promotion of plants inoculated with phosphate solubilizing fungi. Advances in Agronomy 69:99-151.

Xiurong W, Qiang P, Fengxian C, Xiaolong Y, Hong L (2011). Effects of co-inoculation with arbuscular mycorrhizal fungi and rhizobia on soybean growth as related to root architecture and availability of $\mathrm{N}$ and P.Mycorrhiza21(3):173-181.

Zaidi A, Khan MS (2005). Interactive effect of rhizospheric microorganisms on growth yield and nutrient uptake of wheat. Journal of Plant Nutrition 28(12):2079-2092.

Zaidi A, Khan MS, Amil M (2003). Interactive effect of rhizotrophic microorganisms on yield and nutrient uptake of chickpea (Cicer arietinum $\mathrm{L}$ ). European Journal of Agronomy 19(1):15-21.

Zhang F, Dashti N, Hynes RK, Smith DL (1996). Plant growth promoting rhizobacteria and soybean [Glycine max (L.) Merr.] nodulation and nitrogen fixation at suboptimal root zone temperatures. Annals of Botany 77(5):453-460. 